\section{The effect of payoffs and cue tones on detection of sinusoids of} uncertain frequency*

\author{
M. J. PENNER \\ University of California, San Diego, La Jolla, California 92037
}

\begin{abstract}
Previous Es, attempting to distinguish between the single and multiple band models of detection, have found that for some $S s$ the effective listening band is narrow and for others it is not. As in previous experiments, we ask Ss to detect sinusoids of various frequencies in a continuous background noise. However, we influence detection patterns by presenting cue tones and varying payoffs. In some conditions, the $S$ 's detection performance as a function of signal frequency is so peaked that we can estimate the critical bandwidth. In other conditions, the same Ss produce flat detection contours. We interpret these different detection patterns as reflecting changes in the S's listening strategy. It is argued that differences in listening strategy are responsible for the conflicting results found in earlier studies.
\end{abstract}

\section{INTRODUCTION}

In 1940 , Fletcher demonstrated that a continuous pure tone signal in white noise was masked only by the nearby noise frequencies. He called the set of masking frequencies the critical band and postulated that the frequency dimension of the auditory system consisted of a sequence of fixed filter-like mechanisms with bandwidths equal to the widths of the empirically established critical bands. However, Fletcher's discussion is limited to detection of a signal whose frequency was fixed.

An extension of Fletcher's formulation is necessary if a $S$ is asked to detect one of two different frequency signals that are randomly presented. For example, when detecting a $1000-\mathrm{Hz}$ tone, only the noise in a critical band about $1000 \mathrm{~Hz}$ masked the signal. When detecting a $2000-\mathrm{Hz}$ tone, only the noise in a critical band about $2000 \mathrm{~Hz}$ masks the signal. If the signal is equally likely to be $1000 \mathrm{~Hz}$ or $2000 \mathrm{~Hz}$, how will the system perform? It is possible that two filters would be used simultaneously (the multiple band model) or that one filter would be moved rapidly between the two signal frequencies (the single band model). Neither the single nor the multiple band models based on the width of the critical band can account for the small decrement that is experimentally obtained when the signal frequency changes. Both models are dependent on a measure of the critical band derived from fixed frequency experiments. If the signal frequency varies, the width of the critical band as measured in a fixed frequency experiment may not be relevant.

* This study was supported by Grant Award NS 06940-06 from the National Institute of Health.
There is reason to believe that the $S$ 's bandwidth is variable. For example, in Green's (1960) noise, $650 \mathrm{~Hz}$ wide, presented in a background of continuous noise. The width of the critical band is a function of frequency; it increases as the center the center frequency of the signal increases, more of it falls within a critical band. If the noise signal rises in frequency and if the $S$ could listen to only one critical band, he would be ignoring progressively less of the signal. Hence detectability should improve as the center frequency of the noise signal rises. In fact, Green found that detection was independent of the center frequency of the signal. He concluded that "critical bands" could be adjusted when it was advantageous to do so.

If the system is adjustable, then it is not surprising that models based on fixed critical bandwidths are inconsistent with the data. In fact, if the system is adjustable, then we should examine the parameters that lead to adjustment. There are at least four experimental conditions that might affect the detection pattern of sinusoids of uncertain frequency: deception, probability variation, payoffs, and cue tones.

\section{Deception}

One method that manipulated the him that the signal was fixed in frequency but to vary it experimentally. Under these conditions, it is argued that the $S$ listens with a filter centered about the expected frequency and ought to detect only signals in the critical band surrounding it. Es (Tanner, 1956, as cited in Swets, 1963; Greenberg \& experiment, the signal was a band of frequency of the signal rises. Thus, as S's detection pattern was to convince
Larkin, 1968) have found that signals of remote unexpected frequency are not detected. This does not necessarily mean that the $S$ has narrowed a single filter about the expected frequency and does not hear the off-frequency tones; it may be the the $S$ hears the off-frequency signals and does not report them since they do not "sound like" the usual signal.

\section{Probability Variation}

A second way to change patterns of detection is to vary the a priori probability of presenting a frequency. However, specifying the probability of presenting a particular frequency signal does not define a unique ideal detection pattern. The $O$ who is aware of the a priori probabilities may maximize the percent of correct detections, perform some type of probability matching, or combine strategies. If a single band model $O$ tries to maximize the overall percent correct, he will set his single band about the signal with the highest presentation probability. On the other hand, if an attempt is made to probability match, then the probability of detecting the most frequently presented signal decreases. Similarly, a multiple band 0 could ignore the a priori frequency probability and detect all-signals equiprobably. Alternately, he could weight the filter outputs proportionally to the signal's occurrence (probability matching). Lastly, he might attempt to maximize the overall probability of a correct response by listening only to the filter in which the signal is most likely to occur. Thus, simply specifying the probability of a signal's frequency does not determine a unique detection pattern. Hence it is not surprising that varying the a priori probability of a signal's frequency (Sorkin et al, 1969) has not radically affected detection. However, probability variation may be a reasonable approach to manipulation of detection scores if coupled with more explicit instructions.

\section{Payoffs}

A third way to change the pattem of detection is to use payoffs. The idea behind the use of payoffs is that if it can be made monetarily important to detect a signal of specific frequency, then detection of signals of other frequencies will be sacrificed.

Larkin and Greenberg (1970) recently found that their payoffs hardly affected differential detectability of a $500-\mathrm{Hz}$ and an $1100-\mathrm{Hz}$ tone. However, their payoff matrix might not have been adequate. For some Ss, multiple band behavior rather than single band behavior would result in a loss of $25 \%$ of the payoff, 


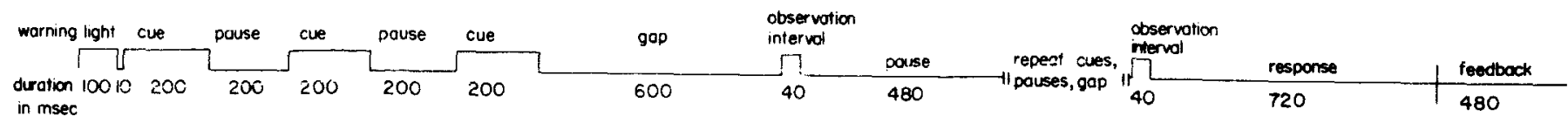

Fig. 1. Schematic diagram of a single 2AFC trial.

or $\$ .25$ per day. This is approximately a $7 \%$ decrease in total daily salary. Such a small differential reward might not be adequate to encourage a multiple band $S$ to listen to a narrow frequency region. The use of a payoff matrix with a large differential reward might, in fact, influence patterns of detection of sinusoids of variable frequency.

\section{Cueing}

A fourth means of manipulating detection patterns is the use of cue tones. If the effect of deception were really due to the filter being centered about the expected frequency and if a cue tone could assist the $S$ in centering his filter, then cue tones would alter detection patterns. The cue tone might eliminate the effect of frequency uncertainty for signals of the same frequency as the cue.

However, uncertain frequency experiments using cue tones (Greenberg, 1961; Swets \& Sewell, 1961) show a decrement in detection even when the cue and test tones are the same frequency. There are several reasons why this might be. In Greenberg's experiment the cue tone was barely audible; the probability of detecting it was 0.75 . In Swets and Sewall's experiment the cue tone was not identical to the test tone since the noise was attenuated. It might be, therefore, that the probability of detecting a test tone in an uncertain frequency experiment with a sufficiently long cue tone would be equal to the probability of detecting a test tone in a 2AFC task with no frequency uncertainty.

In summary, detection patterns may be influenced by at least four methods: deception, probability variation, payoffs, or cue tones. Deception is an unsatisfactory means of manipulation since it is unclear what the $S$ actually heard. Varying the probability of a signal frequency does not determine an ideal unique detection pattern. The effect of payoffs and cue tone have not been thoroughly investigated. The following experiment uses both payoffs and cue tones to shift the pattern of uncertain frequency detection. Thus, we shall determine if a combination of payoffs and cue tones affect detection patterns.

\section{PROCEDURE} Apparatus

The experiment was controlled by a PDP-8 computer. The cue tones and the pure tone signal (produced by a programmable Wavetek, Model 155) were presented in a continuous background noise (General Radio noise generator, Model 1388). The signals were gated on at the positive going zero crossing, attenuated, mixed with the noise, and then sent to a soundproof booth (IAC, Model 1202A) where the Ss heard the signals through TDH-39 earphones. Three Ss were in the booth simultaneously.

\section{Paradigm}

The basic experimental paradigm is diagrammed in Fig. 1. The procedure was 2AFC with three identical cue tones presented before each observation interval, with a 720-msec response interval, and with feedback lasting $480 \mathrm{msec}$. Each trial was preceded by at 100 -msec warning light followed by a 10-msec pause. Each half of the 2AFC presentation contained three identical 200-msec cue tones separated by $200 \mathrm{msec}$, a 600-msec gap time followed by a 40-msec observation interval. The cue and test tone durations were selected on the basis of a pilot experiment. There were two separate neon lights each marking an observation interval. The feedback consisted of turning on the neon light which had marked the interval in which the signal had occurred. Immediately following the offset of the feedback light a new trial began. The Ss responded by pushing the toggle switch below the light which they believed to have marked the interval in which the signal occurred. The noise was continuous.

Signal-to-noise ratios were adjusted according to Green et al (1959), and are reported in the figure captions. Thresholds were obtained for each signal frequency so that Ss would be familiar with all possible signals. There was a minimum of 600 observations for each signal frequency in the fixed frequency condition.

Three sets of frequencies were presented. In one, the cue tone was always $1500 \mathrm{~Hz}$, and the possible test tones were $1200,1300,1400,1440$, $1500,1560,1600,1700$, or $1800 \mathrm{~Hz}$. In the second test, the cue tone was always $500 \mathrm{~Hz}$, and the possible test tones were $300,410,470,500,560$, 590 , or $700 \mathrm{~Hz}$. For the third, the cue tone was always $4500 \mathrm{~Hz}$; the possible test tones were $3600,3900,4200$, $4500,4800,5100$, or $5400 \mathrm{~Hz}$. The test tones were all presented with equal probability.
Payoffs

There were two payoff conditions used to affect the S's strategy. In both conditions, the competitiveness of the Ss was enlisted to increase the effectiveness of a monetary payoff. In Condition A, $\$ .25$ was paid to the $S$ whose probability, $P_{Q}$, of detecting the test tone when it was the same frequency as the cue tone came closest to or exceeded the probability, $\mathrm{O}_{\mathbf{Q}}$, of detecting the test tone when it was the only frequency signal presented in a $2 A F C$ task [payoff for $\left.\max \left(P_{Q}-O_{Q}\right)\right]$. The subscript $Q$ indicates that the test tone is the same frequency as the cue. Thus, this payoff essentially instructed the Ss to attend to the test tone having the same frequency as the cue tone. In addition, $\$ .10$ was paid to the $S$ with the highest overall probability of detection relative to his prior detection scores (payoff for maximum

$$
\left[\sum_{\mathbf{i}}\left(\mathbf{P}_{\mathbf{i}}-\mathbf{O}_{\mathbf{i}}\right)\right],
$$

i represents frequency). This last payoff was intended to motivate the $\mathrm{Ss}$ to report all the signals they heard even when the test tone was not the same frequency as the cue tone. Note that these payoffs are not mutually exclusive; one $S$ could win both. In Condition B, Ss were told to attempt to detect all frequencies. To encourage this, $\$ .25$ was paid to the $S$ with the most nearly equal detection scores (payoff for $\mathrm{P}_{i}-\mathrm{O}_{i}=\mathrm{k}$ ) for every frequency presented. In addition, $\$ .10$ was paid to the $\mathbf{S}$ whose probability of detecting the test tone when it was the same frequency as the cue tone, came closest to, or exceeded the probability of detecting the test tone when it was the only frequency signal in a $2 \mathrm{AFC}$ task [payoff for maximum $\left.\left(P_{Q}-O_{Q}\right)\right]$. This latter payoff was expected to prevent $a$ S from equalizing his detection scores by not reporting the test tone which was the same frequency as the cue. Thus, Ss had the opportunity to win $\$ .35$ per block of trials. Since there were three blocks per day, the total daily payoff was $\$ 1.05$, or $29 \%$ of the S's daily salary without the payoff.

\section{Data Collection}

The data were collected in the following order: practice with fixed signal frequencies, Condition $A$ for the 1500-Hz cue tone, Condition $B$ for the $1500-\mathrm{Hz}$ cue tone; practice with fixed signal frequencies, Condition $B$ for the 

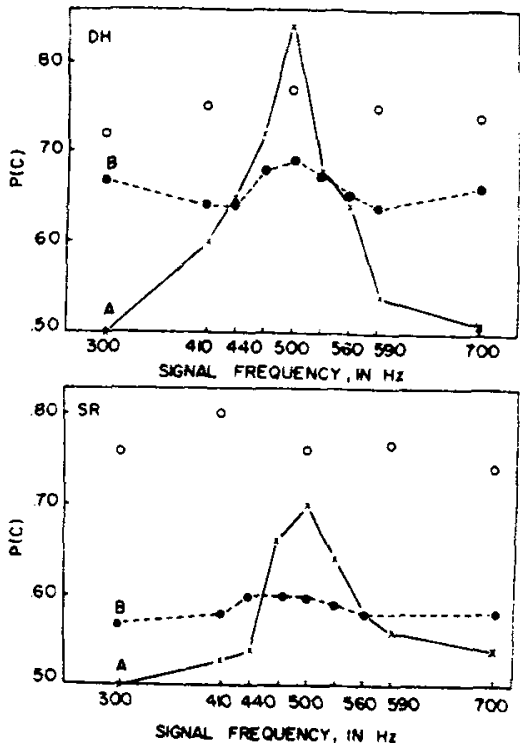

Fig. 2. Graph of the probability of a correct response, $P(C)$, as a function of

500-Hz cue tone, Condition A for the $500-\mathrm{Hz}$ cue tone; practice with fixed signal frequencies, Condition $A$ for the $4500-\mathrm{Hz}$ cue tone.

\section{RESULTS}

The results are presented in Figs. 2 , 3 , and 4 . The data from Condition $A$ are similar to the frequency response characteristic of a filter centered about the frequency of the cue tone, and thus the results might be used to support a single narrow-band model of detection. If the $S$ centered a filter about the cue tone frequency, then the chance level of detection of signals far removed from the cue tone would indicate that those signals are so greatly attenuated that they are not audible. If these remote signals were detected, the Ss should have reported them in an attempt to increase their payoff.

Assuming that the data of Condition $A$ result from a filter centered about the cue tone frequency, it is possible to estimate the half-power points of the filter. Since $d^{\prime}$ is directionally proportional to $10 \log E_{S} / N_{0}$, d $/ 2^{\prime}$ is proportional to the half-power points of the filter. The two frequencies corresponding to the half-power points provide an estimate of the width of the critcal band. If the filter were centered at $500 \mathrm{~Hz}$, the bandwidths are estimated to be 100,90 , and $80 \mathrm{~Hz}$. Fletcher's (1940) estimate was $51 \mathrm{~Hz}$; Zwicker et al (1957) estimated the width to be $110 \mathrm{~Hz}$. If the filter were centered at $1500 \mathrm{~Hz}$, the bandwidths are estimated to be 150,150 , and $175 \mathrm{~Hz}$. Fletcher's (1940) estimate was $80 \mathrm{~Hz}$; Zwicker et all (1957) estimated the width to be $225 \mathrm{~Hz}$. If the filter were

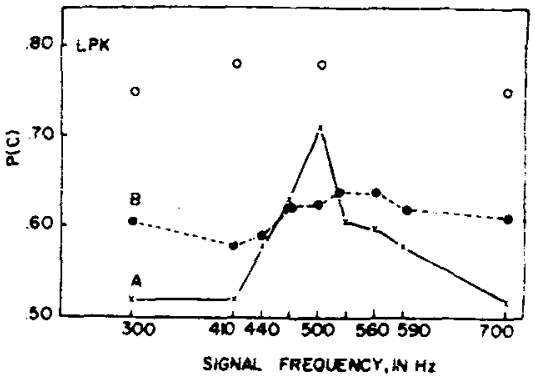

signal frequency for the $500 \cdot \mathrm{Hz}$ tone. The unfilled circles represent $P(C)$ in a 2AFC paradigm with no frequency uncertainty. The xs represent $P(C)$ for Condition $A$, and the filled circles represent $P(C)$ for Condition $B$. For these data, $10 \log \left(E_{s} / N_{0}\right)$ is, in order of increasing frequency, 8.5, 8.6, 8.65 $8.70,9.1,9.13,9.16,9.25,9.60$, and for the cue tone, 9.4. For Conditions $A$ and $B$, the average number of observations per point is 267. The noise power per cycle is $46 \mathrm{~dB}$ SPL.

centered at $4500 \mathrm{~Hz}$, the bandwidths are estimated to be 830,900 , and $1050 \mathrm{~Hz}$. Fletcher's estimate was about $250 \mathrm{~Hz}$; Zwicker et al estimated the width to be about $750 \mathrm{~Hz}$. All but three of the widths of the critical band estimated in this experiment are thus within the usual range.

However, to reach a reasonable level of confidence about these estimates of the width of the critical band, many
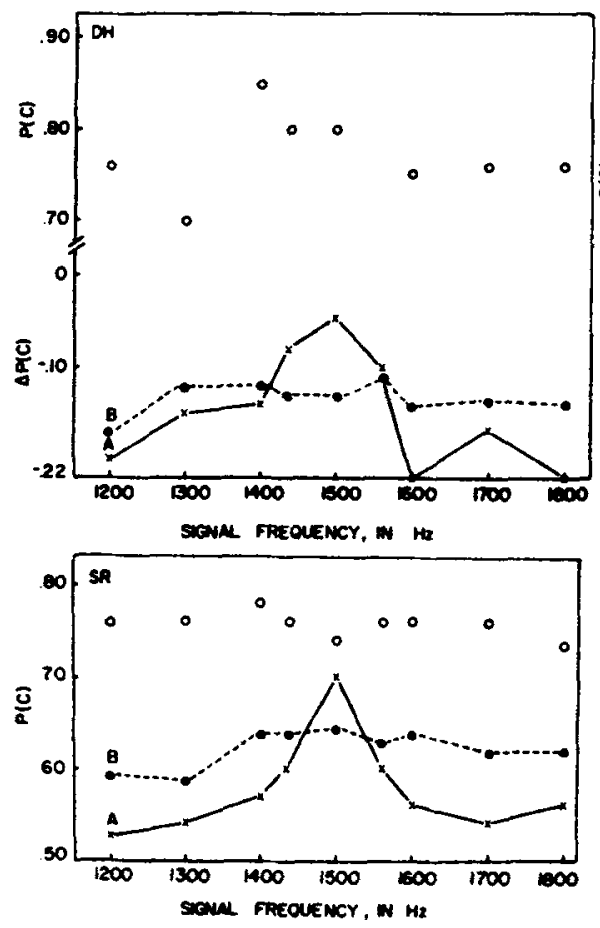

Fig. 3. Graph of the probability of a correct response, $P(C)$, as a function of signal frequency for the $1500-\mathrm{Hz}$ tone. measurements would be required. A slight variation in detection scores results in sizable changes in estimates of critical bandwidth. For example, if SR's maximum level of detection were 0.71 rather than 0.68 , the width of the

critical band about $1500 \mathrm{~Hz}$ would be 120 rather than $150 \mathrm{~Hz}$. Neverthless, the obtained critical bandwidth estimates are reasonable.

A comparison of Conditions $A$ and $B$ illustrates the effect of payoffs upon detection pattern in uncertain frequency experiments. The results of Condition B are presented in Figs. 2, 3 , and 4 . The data no longer resemble the frequency response characteristic of a single filter. They are instead similar to the data which are well described by the multiple band model, by a rapid scanning single band model, or by a stationary but randomly placed single band model.

\section{DISCUSSION}

From the divergence of the curves produced by Conditions $A$ and $B$ it appears that two very different types of detection patterns can occur. Ss can listen to either narrow or broad frequency regions. Since the detection pattern obtained is dependent upon payoffs, neither pattern can represent an invariant characteristic of the peripheral filtering mechanism. Let us summarize this observation by saying that the $\mathbf{S}$ chooses to detect uncertain

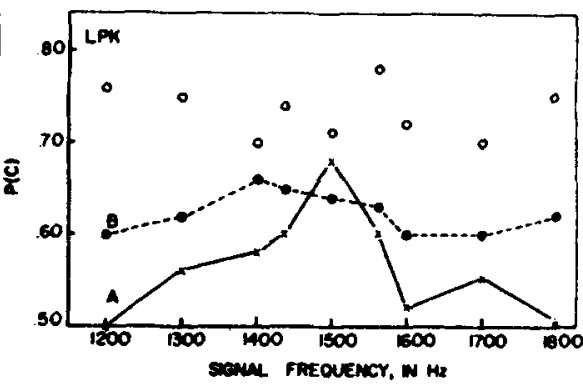

The unfilled circles represent $P(C)$ in a 2AFC paradigm with no frequency uncertainty. The xs represent $P(C)$ for Condition $A$, and the filled circles represent $P(C)$ for Condition B. For $S$ DH we have graphed the change in the probability of detection in the uncertain frequency experiment relative to the fixed frequency case. The change, $\Delta P$, rather than the probability of a correct response is plotted because the range of detection scores, 0.70 to 0.85 , is large. For these data, $10 \log \left(E_{s} / N_{0}\right)$ is, in order of increasing frequency, 11.0, 11.5, 11.9, $12.1,12.3,12.4,13.0,13.3,13.4$, and for the cue tone, 12.6. For Conditions $A$ and $B$, the average number of observations per point is 240 . The noise power per cycle is $46 \mathrm{~dB}$ SPL. 

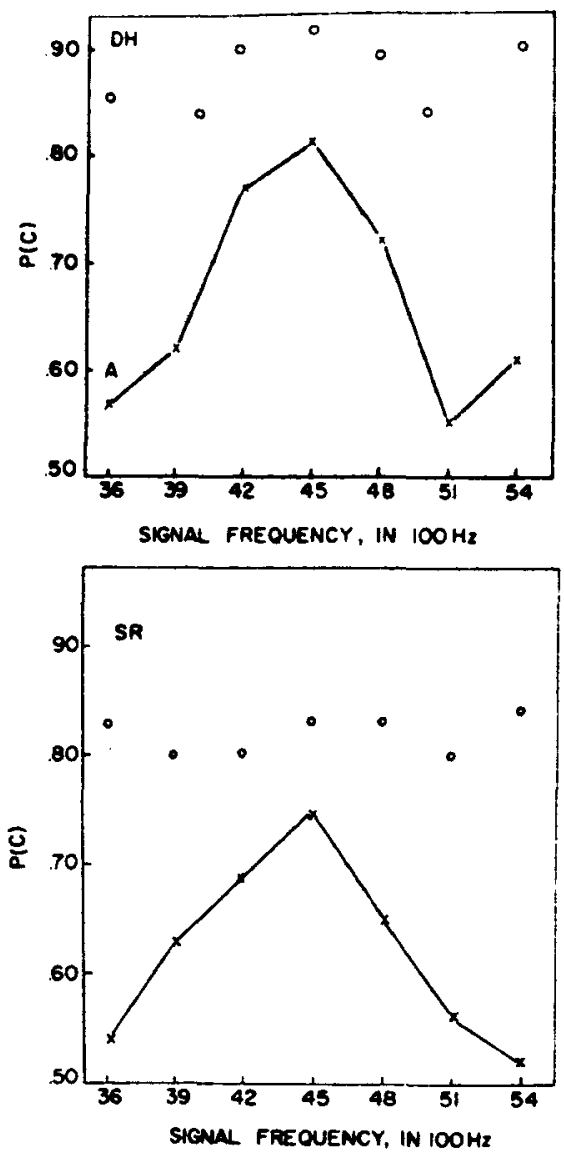

frequencies according to one or another pattern; that is, he selects a listening strategy.

Many previous experiments in frequency uncertainty have not used cue tones. However, detection patterns differ greatly and may have been influenced by strategies induced by implicit instruction. For example, Greenberg and Larkin (1968) obtain a detection pattern similar to Condition A whereas Green's (1961) is similar to Condition B. Greenberg and Larken (1968-see section on deception), by convincing the Ss that only one frequency signal is present, are instructing $S s$ to behave as single-band observers. Before each block of trials, Green (1961), on the other hand, played the lowest, highest, and middle frequency in the range. This might be an implicit instruction to the $S$ to listen to the entire range, and therefore a pattern similar to Condition B is obtained. Implicit instructions are not, however, necessarily sufficient. Ss might use "personal" instructions. Veniar (1958a) remarked that a $S$ who had detected a noise signal in a background of wide-band noise tended to behave as a multiple band observer (produced flat detection contours analogous to Condition B). Zwicker et al (1957),

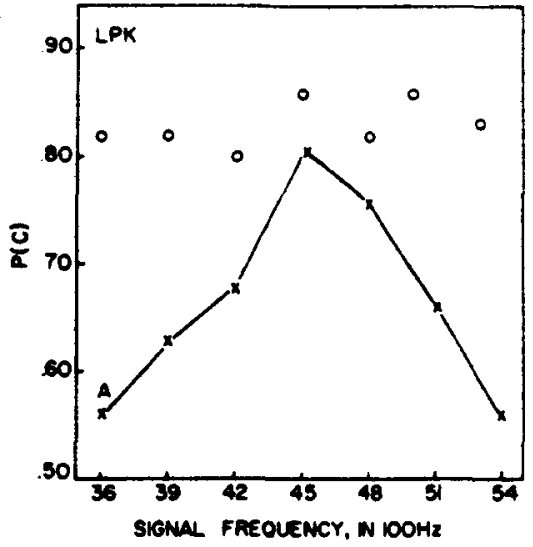

Fig. 4. Graph of the probability of a correct response, $P(C)$, as a function of signal frequency for the $4500 \cdot \mathrm{Hz}$ cue tone. The unfilled circles represent $P(C)$ in a $2 A F C$ paradigm with no frequency uncertainty. The xs represent $P(C)$ for Condition $A$. For these data, $10 \log \left(E_{s} / N_{0}\right)$ is, in order of increasing frequency, 14.1, 14.2, $14.3,14.4,14.6,14.7,14.9$, and for the cue tone, 14.7. For Condition $A$, the average number of observations per point is 334 . The noise power per cycle is $46 \mathrm{~dB}$ SPL.

noted that some Ss tended to be "analytic"-narrow band-and some tended to be integrative-broad band. More recently, Swets and Kristofferson (1970) reported that Ss tended to behave consistently as either single or multiple band observers in successive psychophysical tasks. This comment illustrates an important point: the effect of instructions on detection patterns of sinusoids of uncertain frequency has traditionally been overlooked.

Listening strategy might be important if data are collected by presenting the same signal in blocks of trials rather than randomizing the signal parameters from trial to trial. For example, Leshowitz and Wightman (1970) showed that in order to maximize the signal-to-noise ratio, Ss would detect energy in the side lobes of short duration signals. In any one block of trials, Ss had to listen either to the side lobes or to the main lobe of the pure tone signal. Thus, before each trial, the $\mathbf{S}$ knew the frequency position of the region of maximal signal-to-noise ratio. There is, therefore, no way to determine how quickly $S_{6}$ could adjust and maximize the signal-to-noise ratio because the strategy was predetermined for each block of trials.
Studies of integration time have also been randomized between blocks. Green, Birdsall, and Tanner (1957) found that if the signal enerpy is constant, detection scores are constant for signals ranging from 10 to 150 msec. The signal duration was the same for each block of trials. Thus, for each signal duration the $\mathbf{S}$ could set his bandwidth to the appropriate width for optimal signal detection. If the $S$ could not anticipate the appropriate bandwidth because of trial by trial randomization of signal duration, detection scores might not have been constant throughout the 10 to $150-\mathrm{msec}$ range. Again, listening strategy has been overlooked by randomizing the signal duration from block to block rather than from trial to trial. It would be fascinating to know if the bandwidth of the filter is adapted to the randomized signal duration.

In conclusion, this experiment illustrates that, under certain experimental conditions, Ss operate as if they were listening with a single narrow filter at the cue tone frequency. Under other conditions, they do not. Thus, in this paradigm a choice of listening strategies is available.

\section{REFERENCES}

CREELMAN, C. D.. Detection of signals of uncertain frequency. Joumal of the Acoustical Society of America, 1959, 32 . 805-810.

FLETCHER, H., Auditory patterns. Review of Modem Physics, $1940,12,47-61$.

GREEN, D. M., Detection of multiple component signals in noise. Journal of the Acoustical Society of America, 1958, $30,904.911$.

GREEN, D. M.. Auditory detection of a noise signal. Joumal of the Acoustical Society of America, 1960,32, 121-131.

GREEN, D. M., Detection of auditory sinusoids of uncertain frequency. Journal of the Acoustical Society of America, $1961,33,897-903$.

GREEN, D. M. BIRDSALL. T. G.. \& TANNER, $W, P$.. Jr. Signal detection as a function of signal intensity and duration. Journal of the Acoustical Society of America, 1957, 29, 523-531.

GREEN, D. M., MCKEY, M. J., \& LICKLIDER, J.C. R. Detection of a pulsed sinusoid in noise as a function of frequency. Joumal of the Acoustical Society of America, 1959, 31, 1446-1452.

GREEN, D. M., \& SWETS., J. A. Signal detection theory and psychophysics. New Yodk: Wiley, 1966

GREENBERG, G, Z., Cueing signals and frequency uncertainty in auditory detection. Indiana University: Hearing and Communication Laboratory Technical Report AF 19(628)-266 Fechnical Report

GREENBERG, G. Z.. \& LARKIN, W. D. Frequency-response characteristic of auditory observers detecting signals of a single frequency in noise: The probe-signal method. Joumal of the Acoustical Society of America, 1968, 44, $1513-1523$.

LARKIN, W. D., \& GREENBERG, G. $Z$. Selective attention in uncertain frequency detection. Perception \& Psychophysics, 1970. 8, 179-184. 
LESHOWITZ, B.. \& WIGHTMAN, F. L. On-frequency masking with continuous sinusoids. Journal of the Acoustical Society of Amexica, 1971, 49, $1180-1190$.

SORKIN, R. D., PASTORE, R. E., \& GILLIOM, J. D. Signal probability and the listening band. Perception \& Psychophysics, 1968, 4, 10-12.

SWETS, J. A. Central factors in auditory frequency selectivity. Psychological
Bulletín, 1963. 60, 429-440.

SWETS, J. A., \& KRISTOFFERSON, A. B. Attention. Annual Revieu of Psychology. $1970,21,339$.

SWETS, J. A.. \& SEWELL, S. T. Stimulus versus response uncertainty in recognition. Journal of the Acoustical Society of America, 1961, 33. $1568-1592$.

VENIAR, F. A. Signal detection as a function of frequency ensemble, I.
Journal of the Acoustical Society of America. 1958a, 30, 1020-1024.

VENIAR. F. A. Signal detection as a function of frequency ensemble, II Joumal of the Acoustical Society of America, 1958b, 30,1075-1078.

Z WICKER, E.. FLOTTORP, G., \& STEVENS. S. S. Critical bandwidth in loudness summation. Journal of the Acoustical Society of America, 1957, 29, 548-557. 